\title{
Body motion, early algebra, and the colours of abstraction
}

\section{Ricardo Nemirovsky ${ }^{1}$ (D) Francesca Ferrara $^{2} \cdot$ Giulia Ferrari $^{2}$. Natividad Adamuz-Povedano ${ }^{3}$}

Published online: 8 June 2020

(C) The Author(s) 2020

\begin{abstract}
This paper focuses on the emergence of abstraction through the use of a new kind of motion detector-WiiGraph-with 11-year-old children. In the selected episodes, the children used this motion detector to create three simultaneous graphs of position vs. time: two graphs for the motion of each hand and a third one corresponding to their difference. They explored relationships that can be ascribed to an equation of the type $\mathrm{A}-\mathrm{B}=\mathrm{C}$. We examine the notion of abstraction on its own, without assuming a dualism abstract-concrete according to which more of one is less of the other. We propose a distinct path for the attainment of abstraction, which involves navigating a surplus of sensible qualities. The work described in this paper belongs to early algebra, we suggest, because it involves the elementary symbolic treatment of unknowns and generals. More broadly, it advances a perspective on the nature of mathematical abstraction.
\end{abstract}

Keywords Early algebra $\cdot$ Abstraction · Graphing motion - Sensorimotor qualities · Semiosis · General

Ricardo Nemirovsky

r.nemirovsky@mmu.ac.uk

Francesca Ferrara

francesca.ferrara@unito.it

Giulia Ferrari

giulia.ferrari@unito.it

Natividad Adamuz-Povedano

nadamuz@uco.es

1 Manchester Metropolitan University, ESRI, Manchester, UK

2 Università di Torino, Torino, Italy

3 Universidad de Córdoba, Córdoba, Spain 


\section{Introduction}

Learning mathematics is often seen as a progression or movement from the concrete to the abstract. This progression amounts to a passage across emphases, from the sensible to the intelligible. An archetypal example is that of the straight line. Out of countless acts of drawing, touching straight edges, tracing on the sand, or using tools, a sense grows for physical straightness. There is still a major gap between the latter and a geometric straight line involving a massive drawing out of sensible qualities, such as colour, length, material, and thickness, to envision an entity that is intelligible but not sensible. Hence abstraction is depicted as a subtractive process, along which more and more qualities are taken out until a spectral remainder is left that is not amenable to being touched, seen, or heard and is devoid of causal powers, whose presence is only indirectly evoked by diagrams and formulae.

Figure 1 is an attempt to illustrate this vision for the obtainment of abstraction. It is necessarily paradoxical because in this approach, no perceivable entity can display an abstraction as such. The figure includes a rectangle at the bottom appearing to be a blackboard full of marks chalked up on it and a closed curved line above. The rectangle at the bottom symbolizes a plane encompassing sensorimotor qualities of objects, organisms, and symbols (e.g., colours, textures, and sounds), and the shape above is an abstraction that lacks sensorimotor qualities, even though its displaying a particular shape, size, and so on betrays the paradox of portraying an abstraction as visible. The core idea of this approach to the attainment of abstraction is that of a process of transcending planes of sensorimotor qualities, reaching out to an external realm unavailable to perception and causal powers.

Numerous researchers in mathematics education have questioned this traditional image for the attainment of abstraction (Clements, 2000; Dreyfus, 2014; Hershkowitz, Schwarz, \&
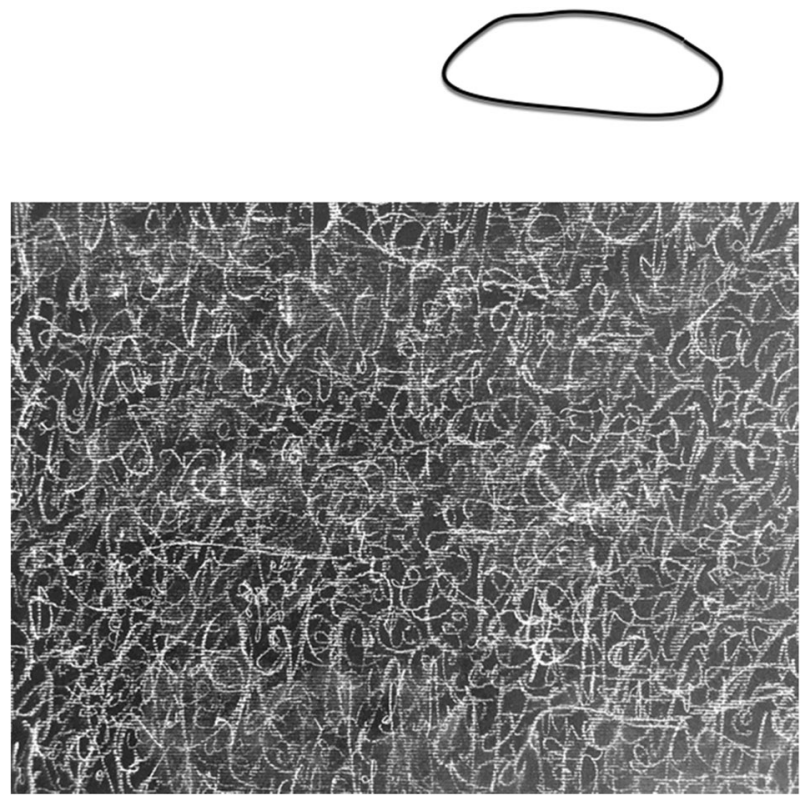

Fig. 1 A vision of abstraction as transcendent to a plane of sensorimotor qualities (Drawing by Giulia Ferrari, white chalk on black cardboard) 
Dreyfus, 2001; Noss, Hoyles, \& Pozzi, 2002; Roth \& Hwang, 2006). Wilensky (1991) argued that ideas are abstract or concrete depending on how thinkers relate to them. Someone practiced with linear equations, for instance, might sense a concreteness in them that is unavailable to someone unfamiliar with them, for whom they are abstract. From this point of view, abstraction is a deficient mode, and the learning of mathematics is rather a progression from the abstract to the concrete, which he called "concretion." Clements (2000) pointed out that the roots of the word "concrete" lie in the idea of growing together and introduce two kinds of concrete knowledge: sensory-concrete and integrated-concrete. The first implies the use of sensory material in the process of sense making; the second combines ideas towards a new structure and implicates physical and abstract knowledge. Noss et al. (2002) introduced the notion of "situated abstraction" seeking to describe how a conceptualization of mathematical knowledge can simultaneously implicate both, the specificity of a situation and the generality of an abstraction, in a way that these two aspects are interwoven and can feed one another. Hershkowitz et al. (2001) proposed to think of "abstraction in context" to avoid a description of abstraction as some type of decontextualization - akin to our radical "subtraction process"- which they find in most cognitivist approaches. Abstraction in context is an activity or process of reorganization of previous mathematical knowledge into new mathematical structures that incorporate the context motivating it. Coles and Sinclair (2018) critique the assumption that learning should begin with the concrete and familiar while abstraction arrives later. They argue for a relational view according to which number learning is, from the very beginning, rooted in abstractions that are already part of young children's lives. Roth and Hwang (2006) analysed a "think aloud" interview with an ecologist as he made sense of a graph he had not used previously. On the basis of a microanalysis of utterances and gestures, they conclude that "rather than being a movement from concrete to abstract or from abstract to concrete, development occurs in a movement that appears to be simultaneously from concrete to abstract and from abstract to concrete" (p. 318). These perspectives seem to, partially at least, resonate with Davydov (1990) epistemological theory considering a dialectical connection between abstract and concrete. According to Davydov, there are two types of abstraction: empirical and theoretical. An empirical abstraction involves the isolation of a certain perceivable quality common to a set of instances; a theoretical abstraction is organized around theoretical models interrelating unperceivable features participating in the genesis or formation of members of a certain class.

Drawing on some of the work reviewed above, we move in this paper towards conceptions of abstraction that (1) examine the notions of abstract and concrete on their own instead of secluding them into a confining dualism, according to which more of one is less of the other, and, (2) rather than a movement away from sensorimotor qualities, portrays the activity of abstracting as navigating or wayfaring a plane of sensorimotor qualities: abstraction as immanent to a plane of sensorimotor qualities. Figure 2 is an attempt to illustrate this second point. In contrast to Fig. 1, the shape indicating an abstraction is in a plane of sensorimotor qualities, not as an object but as a navigational path, like a trail maintained by numerous "walks" - the added arrow intends to show this wayfaring quality.

This paper expresses an effort to flesh out this vision of abstracting as navigating a plane of sensorimotor qualities. In order to unfold grounds for this fleshing out, we have selected three critical concepts to elaborate further in the next section: (1) sense and reference: on the contents of a plane of sensorimotor qualities, (2) generals and unknowns: on different ways of navigating planes of sensorimotor qualities, and (3) semiosis: on flows and temporality in a plane of sensorimotor qualities. 


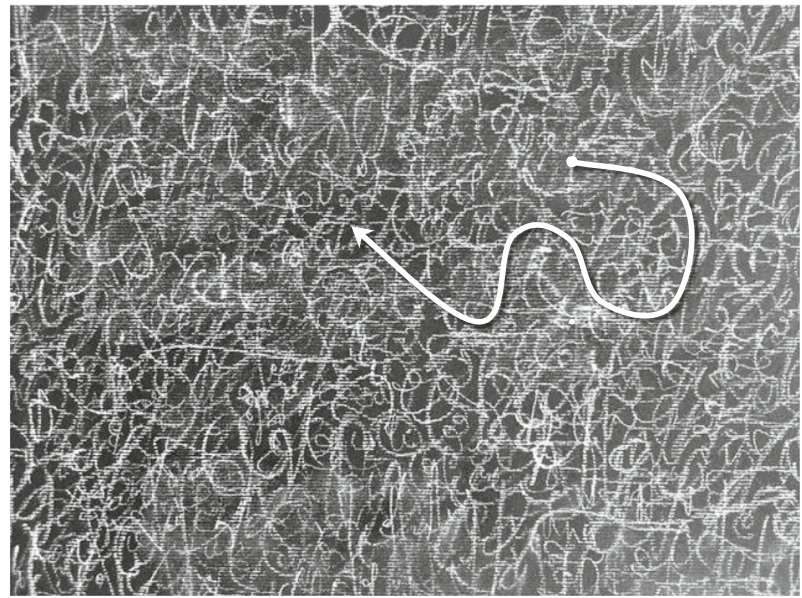

Fig. 2 A vision of abstraction as immanent to a plane of sensorimotor qualities

\section{Theoretical framework}

\subsection{Sense and reference: on the contents of a plane of sensorimotor qualities}

In 1892, Frege published one of his most influential papers whose translated title is "On Sense and Reference" (Frege, 1980). In this paper, he illustrated the distinction with the famous example of Venus: the planet is the evening star (i.e., Venus becomes visible first after the sunset) and morning star (i.e., Venus stays visible last after sunrise); the idea being that the same referent, Venus, can be referred through different senses, such as the "morning star" and the "evening star." Enacting different senses for the same reference is pervasive in linguistic interactions. We easily understand, for instance, of someone called Mary, that "Mary is a baseball player" and "Mary is a dedicated student" convey two different senses for the same person. In general, a proposition pinpoints a referent by means of a certain sense, chosen among multiple senses that are possible for it. This is true of algebraic propositions as well. For example, in reference to the following quadratic equation:

$$
y=2 x^{2}+x+1
$$

It can be said that "Eq. (1.1) has complex roots" and "Eq. (1.1) describes a motion with constant acceleration." These two different senses of the same equation can be more or less significant depending on the situation the speaker is grappling with. To capture this active texture of situation and utterance, it is useful to adopt Deleuze's (1990) approach, thinking of sense as event. We illustrate this aspect by means of an example. The sense expressed by "Eq. (1.1) has two complex roots" might be an event that includes uttering it as part of a problem process calling for the finding of its roots and/or the discrimination of whether they are real or complex. A sense of Eq. (1.1) is a certain problem-solving event. There are no senses without events. Senses emerge out of the way we deal with a situation calling for an event, such as the determination of the roots of Eq. (1.1).

Note that this use of the word "sense" is different from the one customarily invoked in English definitions in biological terms of a physiological capacity of organisms that provides 
data for perception (https://en.wikipedia.org/wiki/Sense), such as vision and touch. Our use is more related to what the phrase "making sense" conveys, conjuring up ideas of meaning or significance. This is essential to avoid flattening planes of sensorimotor qualities onto assemblages of multifarious unarticulated sensations. Although difficult to point out, this work of clarification is intended to be done by the word "qualities." "Mary is a dedicated student" illuminates a particular quality that subsumes or encompasses countless aspects of her life and of sociocultural expectations for what a "dedicated student" is. Each distinct quality (e.g., "Mary is a baseball player") points at an inexhaustible horizon of life events and sociocultural expectations.

Returning to the title of this section, which alludes to the "content of a plane of sensorimotor qualities," we can now assert that such content is made out of interrelated senses, events, and qualities. It follows that navigating such a plane in the course of abstracting entails exploring senses, participating in events, and envisioning or feeling qualities. The sense of Eq. (1.1) pointed at by the proposition "Eq. (1.1) has two complex roots" might turn on a path leading to complex functions in $R^{2} \rightarrow R^{2}$ mappings, to make the roots of Eq. (1.1) as visible as the roots of real functions on a Cartesian graph. Just to add a second example, a request to divide two polynomials might prompt navigating away from many practices engaged by dividing numbers - polynomials do not seem to be something that can be fractionally divided - towards revising sociocultural expectations ordinarily elicited by the word "divide."

\subsection{Generals and unknowns: on different ways of navigating planes of sensorimotor qualities}

Concluding his commentaries about multiple mythical narratives, such as the one of Thales measuring the height of an Egyptian pyramid by the shadow of a stick, or the use of the gnomon in ancient Babylonia, Serres (2017) insists "Yes, its abstraction is a sum and not a subtraction" (p. 210) and introduces the image of white light "Geometry integrates all our practical or ideal habitats the way white light sums up all the colours, in transparency or translucency" (p. 210). This remark has inspired us to think of vast aggregates of senses, events, and qualities - as numerous as colours are in white light - from within which abstractions emerge in the course of their navigation. This opens up questions about kinds of navigation/abstraction that may trailblaze a plane of sensorimotor qualities. We will distinguish two of them: navigating to grasp a general and navigating to solve something vaguely known (i.e., an unknown). In order to clarify what these are, we will first review the distinction, introduced by Peirce, between the general and the vague.

Let us start with the notion of a general:

A sign is objectively general, in so far as, leaving its effective interpretation indeterminate, it surrenders to the interpreter the right of completing the determination for himself.

"Man is mortal." "What man?" "Any man you like." (CP 5.505)

A theorem proving a property of triangles, for example, deals with triangles as a general. A general is genuinely indeterminate. Note that generals are not necessarily produced by generalizations, since the latter involve no more than extending a finite set of empirical observations. "Triangles," as a general, refers to a multiplicity of items that are all actively related to each other through a continuous and mutual communication of differences. In fact, Peirce saw a deep connection between generals and the continuum. Peirce deemed that a general is unlike a finite or infinite set of discrete elements. Even the set of real numbers, customarily used to illustrate the continuity of a line, would not correspond to a continuum 
according to the late Peircean sense, because no matter how many infinite points are added to a set, they still remain in isolation from each other. What truly establishes a continuum is a mutual communication or connectedness that cannot arise from isolated elements, regardless of their numerosity. In contrast to generals, Peirce characterized unknowns - particulars with certain but unspecified traits-as "vague:"

A sign is objectively vague, in so far as, leaving its interpretation more or less indeterminate, it reserves for some other possible sign or experience the function of completing the determination. "This month," says the almanac-oracle, "a great event is to happen." "What event?" "Oh, we shall see. The almanac doesn't tell that." The general might be defined as that to which the principle of excluded middle does not apply. A triangle in general is not isosceles nor equilateral; nor is a triangle in general scalene. The vague might be defined as that to which the principle of contradiction does not apply. For it is false neither that an animal (in a vague sense) is male, nor that an animal is female. (CP 5.505)

We are uncertain whether the eye colour of a friend is green or brown, but we know that it is not, say, red. The vagueness of her eye colour includes infinite shades of brown and green and excludes redness. Together with such vague sense of eye colour, we may also presume that her eyes are of a particular colour, which is the key character of an unknown: its traits are determined but we know them only vaguely.

Grappling with an unknown entails relating to an entity that lacks, perhaps only momentarily, certain sensible qualities both in itself (e.g., her eye colour) or in its signs (e.g., a textual description of her eye colour). On the other hand, we navigate a general, such as mortals or triangles, by immersing ourselves in a vast and familiar terrain of sensible variations and differences, such as mortals of different age, sex, species, bodies, and behaviours or triangles differing in shape, size, angles, perimeters, and colours. The high school problem of determining the length of a side of a triangle, given the length of its other two sides and the angle in between them, is likely to confront us with an unknown, that is, a quality that is only vaguely known (e.g., if two sides are a few centimetres long, the length of the third one is vaguely known to be shorter than a metre). On the other hand, working to demonstrate that, for any triangle, the sum of the lengths of two sides is longer than the length of the third side, whatever one is chosen as the latter, calls us to deal with a general encompassing an infinite number of triangles, not even countable, displaying distributions of infinite possible qualities; unless we prove it by blindly following a scripted sequence of steps, we are likely to be dazzled by the all-embracing universe of entities we are dealing with. Working with generals comprehends all the nuances that pertain to an inexhaustible field we are navigating, while solving an unknown prompts a navigation to a particular destination, from which the unknown may be revealed. Our case study focuses on the former type, that is, on navigating/abstracting to grasp a general. Overall, the question we strive to address in this study is precisely: What kind of navigation towards grasping a general across a surplus of sensorimotor qualities constitutes an abstraction?

We selected episodes in which children explore the kinaesthetic production of graphical expressions, for a general that can be named by the equation: $\mathrm{A}-\mathrm{B}=\mathrm{C}$. We situate our study within the growing field of early algebra (Kieran, Pang, Schifter, \& Fong Ng, 2016). The emphasis of the early algebra work tends to be on the logic of unknowns and on generalizing processes with respect to patterns, variables, structures, and relational thinking (Blanton et al., 2018; Bodanskii, 1969/1991; Carraher, Schliemann, Brizuela, \& Earnest, 2016; Kaput, 2008; 
Kaput, Blanton, \& Moreno, 2008; Ng \& Lee, 2009; Radford, 2014). While marginal, generals are also part of the early algebra literature; Davydov (1990), for instance, proposes ideas that seem to engage children with generals: "In many students even by the end of grade 1 and the beginning of grade 2 ( 8 years) we detected systematic reasoning about rather complex mathematical relations, about their connection, and all of this was done without objects, on a purely verbal level or by relying on letter formulas." (1990, p. 170. Emphasis added). The work described in this paper belongs to early algebra, we suggest, because it involves, directly or indirectly, the elementary symbolic treatment of unknowns and generals.

\subsection{Semiosis: on flows and temporality in a plane of sensorimotor qualities}

Navigating or wayfaring is a dynamic process infused with temporality. We use the concept of semiosis to elucidate this temporal dimension inherent in wayfaring a plane of sensible qualities. Peirce has proposed "semiosis" to mean "an action, or influence, which is, or involves, a cooperation of three subjects, such as a sign, its object, and its interpretant" (CP 5.484). We conjecture that the crucial event (i.e., "action, or influence") of semiosis is a process by means of which a certain quality belonging to a signifier (i.e., sign) expands and begins to incorporate a signified (i.e., referred object) that had previously been unqualified in that way. Semiosis can also proceed in the reverse direction, namely, qualities belonging to a signified begin to newly qualify signifiers. For instance, for an English speaker, the word "smooth" sounds smooth, as if the sound had borrowed such quality from the smooth entities it qualifies, the same for the word "sharp." In these latter examples, semiosis is the historiccultural process through which sounds begin to adopt qualities, such that they come to sound as what they refer to. To a large extent, to become speaker of a certain language involves a complex extension of qualities over thousands of sound patterns or words. Such expansion/ contraction of qualities occurs in a plane of sensible qualities through massive repetition and practice that leaves, over time, a recurrent trace of mutual qualifications for signs/signifiers; these recurrent traces are part of the interpretant of a culture, language, community, or biological species. The interpretant, then, is a continuum sustaining the expansion, contraction, and reproduction of qualities across signifieds and signifiers. Semiosis is never complete; it is always open to uncertain futures. For instance, as one develops a friendship with someone called Manuel, the sound pattern "Manuel" gradually incorporates certain qualities and eschews others expressing expansions and contractions of the qualities one experiences with the friend. This never-ending dynamics shift/expansion/contraction amounts to a form of navigation across a continuum (i.e., the interpretant). In our case study, we will analyse instances in which qualities of a signifier lend attributes to a signified and vice versa, in other words, to the local transformation of an interpretant in response to particular experiences in early algebra.

\section{Methodology}

\subsection{Sensors, kinaesthesia, and mathematical instruments}

In this paper, we attend to the kinaesthetic production of graphical expressions by means of a mathematical instrument. By "mathematical instrument" we refer to a material implement used interactively by means of individual or collective continuous body movements, to obtain and 
transform mathematical expressions, this is related but different from the idea of instrument as discussed in the instrumental approach initially introduced by Verillon and Rabardel (1995), because we want to weaken reference to a psychological characterization of instruments through schemes of usage by the subjects, as we think that it tends to strengthen the dualism separating mental processes from bodily/material actions (Nemirovsky, Kelton, \& Rhodehamel, 2013). We stress continuous body motion: a body does not jump from one spatial configuration to another without traversing interconnected trajectories over time. It is the case that some tools driven by body motion produce discrete sequences, such as texts typed on a computer keyboard, so that the intermediate trajectories between key presses are literally ignored. This is not inherent to keyboards: the performance of any experienced piano player shows that body motion in between key presses fully participates in the musical expression.

Classic examples of mathematical instruments are ruler and compass; other examples are instruments to draw curves, such as ellipses or cycloids. A computer mouse is also an instrument, which in the context of certain software environments may count as mathematical as well, as is the case of dynamic geometry in which dragging becomes a key movement for the tracing of geometric properties (e.g., Baccaglini-Frank \& Mariotti, 2010; Sinclair \& Yurita, 2008; Straesser, 2002). It has been studied in the literature how the fluent use of a mathematical instrument involves the adoption of a "tool perspective" by the users (Nemirovsky, Tierney, \& Wright, 1998). The idea of tool perspective encompasses the emulation of tool's sensitivity to some aspects of an activity rather than others, as well as the recognition of conditions and patterns under which a certain tool-use is significant. We will describe instances of a type of learning situation in which children encounter and productively deal with some algebraic generals through the use of a mathematical instrument we have named "WiiGraph," which was designed by a team led by Ricardo Nemirovsky.

WiiGraph is a graphing motion technology that leverages two hand-held remotes (or Wiimotes) and a LED bar to produce graphical representations. The LED bar has two infrared lights, one on each end. When a Wiimote is pointed at the LED bar, an infrared camera in it "sees" the two infrared lights from the LED bar and, by triangulation, calculates the distance between the Wiimote and the LED bar. In order to make sure that the Wiimotes are pointed to the LED bar - otherwise the infrared lights from the LED bar do not fall within the field of view of the infrared camera in the Wiimote - a large dot is displayed on the computer screen: as long as this large dot is visible on the screen, it indicates that the camera "sees" the infrared lights. We have used a pink and a light blue remote. The pink remote generates a large pink dot and the light blue one a large light blue dot. Under these conditions, when the remotes are moved in front of the LED bar, WiiGraph displays for each of them the position (the distance from the bar) over time, providing two space-time graphs. If a remote is not detected (and the related dot is not visible) for a certain time interval, the corresponding graph is interrupted temporarily and reappears as soon as the infrared lights on the LED bar are seen again from the remote camera. Figure 3 shows the top view and the side view of the interaction space in which a user holds the two remotes and the LED bar is positioned just in front of a large screen. Each line segment in the views marks the distance of a remote from the bar. Note that the remotes do not have to be held at the same height or side position as the LED bar, because as long as the large dots are visible on the screen, the distances are calculated and displayed.

In this paper, we are interested in a specific setting of WiiGraph, which generates in real time an additional third line simultaneously with the two space-time graphs said above. We call it the difference mode, or mode A - B in the following. This third graph corresponds to the difference between the two remotes' distances from the LED bar over time; therefore, it is still 


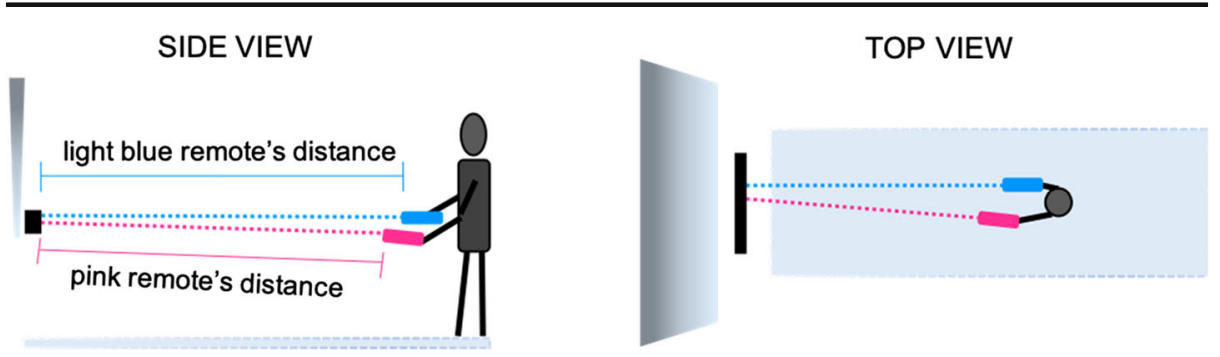

Fig. 3 Top and side view of the interaction space

a distance-time graph displaying the distance between the two remotes in real time. Figure 4 depicts two moments from an experiment using the difference mode, in which a child, Mario, moves the Wiimotes in the interaction space (top) and produces a pink line, a light blue line, and the corresponding difference graph, in dark blue, which is given by pink minus light blue (bottom). The LED bar is located on the edge of the table right in front of the large screen where the graphs are displayed. On the left side, Mario keeps the light blue remote closer to the LED bar than the pink remote; therefore, the pink line is above the light blue line and the difference graph is positive. The right side instead depicts a moment in which the position of the two Wiimotes is exchanged and the pink remote is the closest to the bar, which also means that the difference graph is negative.

WiiGraph works at body scale, that is, involving wider body movements than, for example, those required in moving a mouse, like walking in space or overarm gestures. It displays two movements occurring simultaneously, whether performed by one or two people moving at a time, or two hands, providing continuous feedback to the users. The relationality of the two movements can be expressed in different ways, such as through their difference or ratio. Questions steering investigations with WiiGraph may concern the sustaining of a certain value for these relative ratios or differences over time, as the user interacts by means of body movements. Solutions to these questions end up taking the form of kinaesthetic patterns such as walking with a Wiimote in each
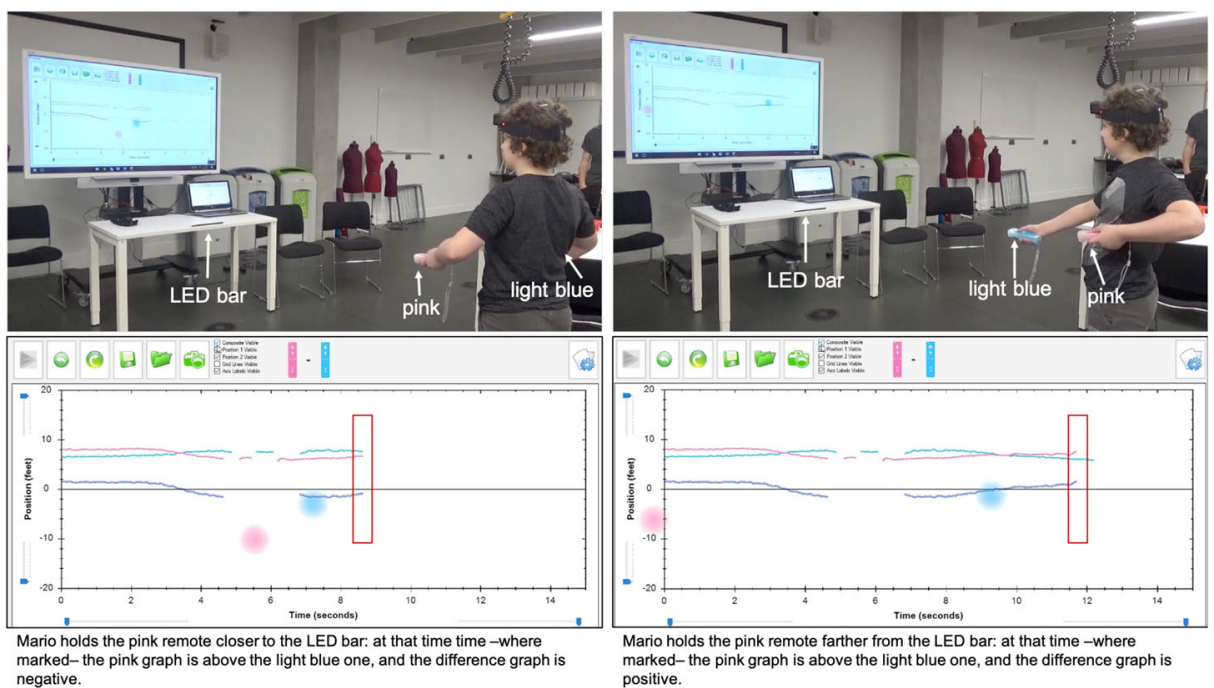

Mario holds the pink remote closer to the LED bar: at that time time -where negative. positive.

Fig. 4 WiiGraph in mode A - B 
hand with a fixed distance between the two Wiimotes. By investigating the use of WiiGraph, we position ourselves in a line of research that values the role of the body and embodiment in mathematical doing, pointing specific attention to the relevance of perceptuo-motor and kinaesthetic activities for approaching and understanding mathematics. It is through these activities that we study the way that abstractions might emerge while navigating sensible qualities involved in WiiGraphing events.

Abrahamson and Sánchez-García (2016) studied the use of an instrument that is similar to the WiiGraph with regard to the users' kinaesthetic engagement. The main thesis of their study is that learning involves "moving in new ways." This can be equally valid for the learning of sports, musical instruments, or mathematics. Given that ways of moving that are formative, say, for playing basketball are not necessarily relevant for playing piano or ping-pong, the question arises: What "new ways of moving" would count as formative to algebra learning? In particular, how do these ways of moving come to mind the gap, often pointed out in the literature, between bodily action and symbolic mathematical activity? Kinaesthetic exploration of generals, such as the one corresponding to the equation $\mathrm{A}-\mathrm{B}=\mathrm{C}$, is our mathematical key to discuss the ways in which minding the gap may occur through the synergy between mathematical instrument and body motion. The roles of motion detectors in mathematics teaching and learning are discussed in a body of literature (e.g., for an overview, see Duijzer, Van den HeuvelPanhuizen, Veldhuis, Doorman, \& Leseman, 2019). However, almost all this literature focuses on the sensing of one moving point. This paper expands the nascent literature on the sensing of two or more moving points, which allow for the exploration of relationalities between them (Abrahamson \& Sánchez-García, 2016; de Freitas, Ferrara, \& Ferrari, 2019).

\subsection{The participants}

We worked with a group of four children aged 11 years, who did not previously know each other, over three sessions. The children had been recruited as volunteers through a network of families practicing home schooling education. Since they do not attend regular lessons at school, we cannot infer their mathematical background. The participants were filmed with two fixed cameras during each session and two of them wore a head-based Go-Pro camera. In addition, we recorded the computer screen with a computer-generated video that later we synchronized with the video from the cameras. The sessions took place at a classroom of a university in England. The conversations with the children were conducted in English. Several of the parents were present in the classroom. During the first two sessions, they explored position vs. time graphs generated by two children, each moving a Wiimote. In addition to free explorations, they engaged in diverse activities anticipating and matching body motions and graphical shapes of position vs. time. In the third session, three children worked by holding both the remotes individually, one remote in each hand. As opposed to a pair of children each handling one Wiimote, the one-in-each-hand arrangement differs markedly, among other reasons because of the centrality it confers to relative arm motion (Nemirovsky, Kelton, \& Rhodehamel, 2012). The instructor chose to turn on the difference graph, as a significant way of exploring relationships between graphs and body motion, beginning the episode we examine in the next section. We have selected this episode because it spans the students' production and exploration of the difference graph. The first and the last author were both present in the classroom. Dan, Mario, and Zev are the names we use for the children. 
The analysis of each transcribed segment is synthesized in its ensuing commentary. We focused our analyses on talk, body motion, gesture, and tool-use. The included commentaries elaborate on those aspects that, we felt, led us to insightful remarks. However, for the sake of completeness and to allow for the belike possibility that readers would develop interpretations that did not occur to us, the annotated transcript describes, as far as possible, all the events that took place, including those that did not elicit an explicit commentary from us.

\section{Selected episode: exploring the difference graph}

This section is an annotated and commented transcription of the episode in which the students experiment with the difference graph. It is divided in two parts. In part one, the students strive to keep the difference graph on the $x$-axis; during the second part, they try to keep the difference graph either above or below the $x$-axis.

\subsection{Introducing the difference graph and trying to keep it on zero}

1. Ricardo: The computer also generates another line [turns on the difference graph] that is, em, dark blue [points at the dark blue graph; Fig. 5a] (...) so we'll investigate what this third line is doing there, what it's showing. So, the first thing we'll try...

2. Mario: It's called, it's called minus because that, that purple [dark blue] line, line is, is, is pink minus blue.

3. Ricardo: OK, how do you know that?

4. Mario: It's real, it's quite obvious, where it says pink minus blue [points to the screen, note the area pointed at with a black arrow in Fig. 5a] at the top of the screen.

5. Ricardo: Aha (...) [gives the two remotes to Mario] so you move, you do whatever you want, [moves alternately right and left hands] but try to keep the dark blue on zero [points to the dark blue line], on this line [left hand runs along the $x$-axis]

Mario begins his first difference graph: he starts with the pink remote in his left hand and the blue one in his right hand. At the beginning of the experiment, the pink remote is kept slightly ahead of the blue one, and then the two are slowly switched in their positions. Holding the two remotes separated, he then walks forward (see the graphs in Fig. 5b).
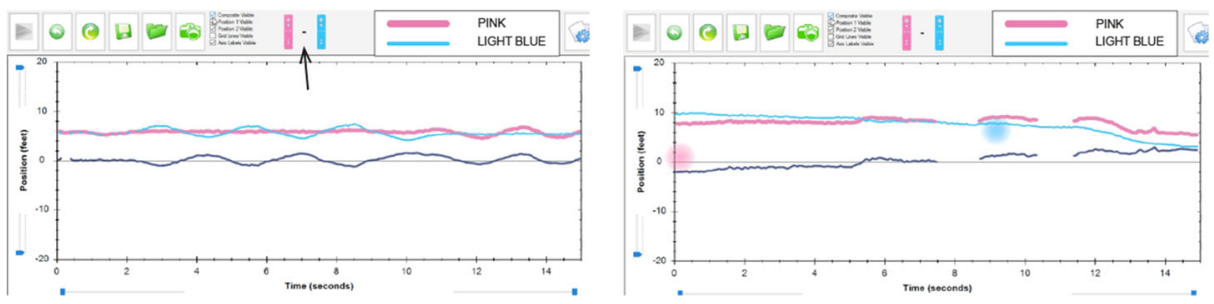

Fig. 5 a Graphical display in which the dark blue difference graph (the darkest in the image) is displayed for the first time. b Mario's first attempt to create a difference graph 

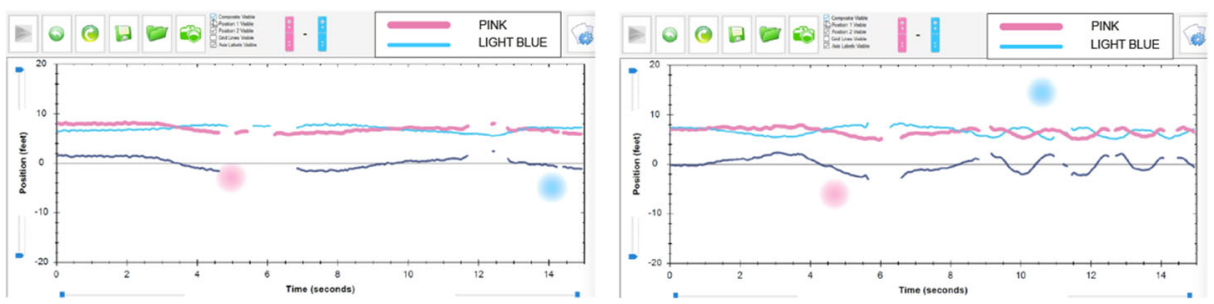

Fig. 6 a Mario's second attempt to create a difference graph. b Mario's new session

During the last seconds of the graph production, he separates the remotes even more and says:

6. Mario: I'm trying as hard as possible not to make the things go opposite.

7. Ricardo: So here you, this piece [pointing to the dark blue graph around second 7] you had it on the line... so try to do more of that, see if you can.

While Ricardo is speaking, Mario moves both remotes back and forth, swinging the arms rhythmically. Then, he starts a new session, moving the remotes slowly in opposite directions, and produces the graphs in Fig. 6a.

During the last seconds, he says:

8. Mario: They're both neutralising each other.

9. Ricardo: Uhum.

10. Mario: That's because, because most of the time I'm, I'm, pink's going in a straight line and blue's going in a stripe, straight line [inaudible] [stops talking, while a new triplet of graphs starts to be created superimposed to the previous one; moves the remotes again back and forth].

Mario presses a button in the Wiimote, and a new session starts: he alternates fluid back and forth movement of the two remotes, which becomes faster and faster (Fig. 6b).

11. Ricardo: So here they were these lines [points to the initial part of the dark blue line in Fig. 6b] ... they, oh, look! [points to the intersection of the dark blue line with the time axis, as the lines unfold] [after second 8, Mario starts to rhythmically bounce on his knees while accelerating the fluid back and forth arm movement creating the second half of the graphs in Fig. 6b]

Commentary The appearance of a third graph prompted Mario to examine the screen seeking for additional signs that could account for it. There was none with a dark blue colour. However, the sign at the top of the screen "pink minus blue," which had been displayed from the beginning of this session but had remained unused, offered him a compelling interpretation ("it is obvious"): the dark blue line "is called minus." The inscription above the graph included the pink and blue Wiimotes, freeing the minus to be clasped by the third graph. The dark blue graph seemed to announce its name. In Paragraph [2], Mario expressed an initial sense for the dark blue graph focused on its name. 
Mario started the graph shown in Fig. 5b with the pink Wiimote in front of the blue one, slowly moving them towards their centre. Once they were next to each other, he continued slowly moving them along the same directions. Right before $8 \mathrm{~s}$, the pink graph disappeared, possibly because the orientation of the pink Wiimote made it fall outside the field of reception. This interruption is likely to have prompted Mario to move the pink Wiimote to make its graph reappear. When it did, the dark blue graph was above the $x$-axis. Then his arms tensed as if trying to push the dark blue graph towards the $x$-axis. Mario reflected on this sense of effort ("trying as hard as possible") as striving "not to make the things go opposite" (Paragraph [6]). This "going opposite" (an event, in Deleuzean sense) might have been the dark blue graph moving in a direction opposite to the desired one, such as towards the zero line. Another possibility, evoked by Mario's use of the plural "things," is that he saw the pink and light blue lines moving in opposite directions, instead of, perhaps, staying together. His reaction was to try to "lower" the pink and blue graphs by walking towards the monitor. However, the dark blue graph continued to inch upwards.

In all his graphical productions (Figs. 5b, 6a and b), Mario tended to move the Wiimotes in alternate directions. This is likely to have followed from tacitly adopting Ricardo's demonstration (see Paragraph [5]). Ricardo gestured an alternate movement of the Wiimotes while saying "you do whatever you want" (Paragraph [5]). While words may leave to the interpretant a more open range of possibilities, gestures are inclined to convey unintended specificities. This tacit assumption of a wavy kinaesthetic pattern was in tension with the task of maintaining the dark blue graph on the $x$-axis. The graph "called minus" was not just a visual display out there but also a curve that resisted physical efforts seeming to possess a will of its own which at times led Mario to tense his movements.

In Paragraph [8], Mario expressed a sense for a general relationship between the pink and blue graphs: "They're both neutralising each other." While his ensuing account of this relationship in Paragraph [10] is inaudible, we hear the sense of Paragraph [8] as indicating the emergence of a general. Recall that dynamic relationships between components affecting each other constitute generals: "neutralizing" suggests a present continuous activity interrelating two graphs or Wiimotes.

Around second 8 of the graphs shown by Fig. 6b, Mario seemed to free himself from trying to keep the dark blue graph close to the horizontal axis, engaging in a new rhythmic kinaesthetic pattern swinging his arms back and forth and bouncing his knees. This bodily movement expressed itself visually by a wavy synchronized variation of the three graphs at once. Relieved from trying to push the dark blue line horizontally, Mario seemed to enjoy a relaxed and smooth swinging - a sense for the graphical weaving emerging on the computer screen as expressed by his wavy body motion.
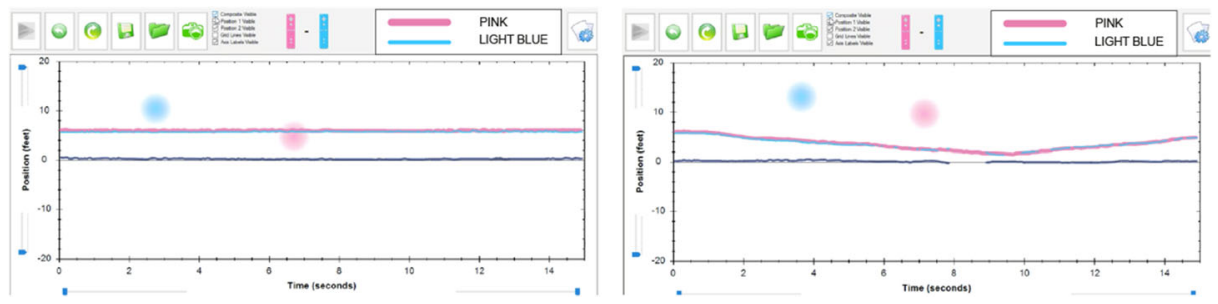

Fig. 7 a Dan generates a graph staying still with the Wiimotes next to each other. b Dan keeps the difference graph on zero while walking 
Mario gives the Wiimotes to Dan, who starts a new graph. He stands still in the same position for all the session, keeping steadily the remotes at the same distance from the LED bar (Fig. 7a).

12. Ricardo: So that, that's a perfect zero! [around the 8th second, laughs] [ending his graph, Dan relaxes his position, shrugs his shoulder and smiles]

13. Ricardo: And, can you do it while you walk?

Dan starts moving very slowly towards the LED bar with both the remotes kept steady and then backwards; he generates the graph shown in Fig. 7b:

14. Dan: You just have to keep the remotes in (...) one position.

15. Ricardo: Like, keeping [them] together?

16. Dan: Keeping them at the same level.

17. Ricardo: The same level, ok.

Dan creates then new graphs, walking again towards the LED bar, then backwards, keeping the remotes steady, next to each other.

Commentary Dan came to create a horizontal difference graph with a clear plan — stay still with the two remotes next to each other - that he had developed while observing Mario's experimentation. He had a well-defined sense that a dark blue graph on the horizontal axis "converted" into the two Wiimotes being next to each other. Moreover, Dan easily showed in Fig. $7 \mathrm{~b}$ that that condition was indifferent to his walking distance from the LED bar: "You just have to keep the remotes in one position" (Paragraph [14]). Dan articulated his sense for the relationship between the dark blue graph being on the $x$-axis and the range of kinaesthetic activities consistent with it in two ways: "keep the remotes in (...) one position" (Paragraph [14]) and "Keeping them at the same level" (Paragraph [16]). While the word "position" alludes to a location in space, the word "level" is customarily a term for height. So far, the children's experimentation with the Wiimotes had not included varying the kinaesthetic quality of the Wiimotes/hands' height, to ascertain graphical responsiveness. On the other hand, differences in height between the light blue and pink graphs had been of major significance. We surmise that Dan's relevance of the Wiimotes being at equal levels had drifted from noticeable graphical levels to the taken-by-default levels of the Wiimotes. This "out reaching" of qualities from one signifier (e.g., graphs' levels) to another (e.g., Wiimotes' levels), which end up encompassing both, is an instance of what we have interpreted in Sect. 2.3 as "semiosis."
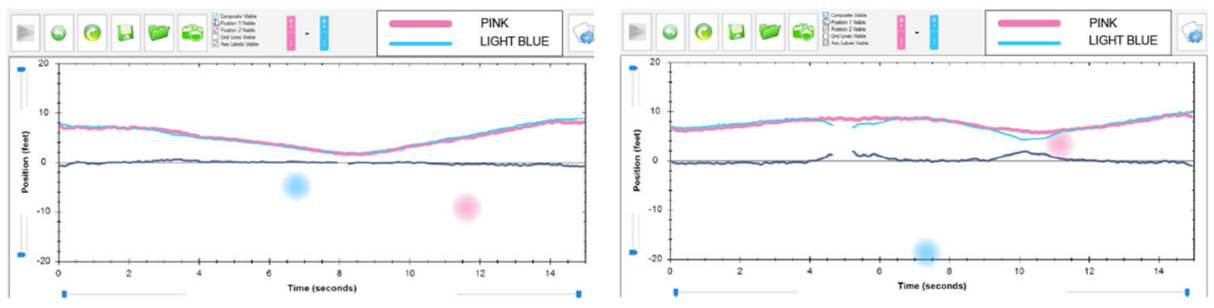

Fig. 8 Zev keeps the difference graph on zero while walking 


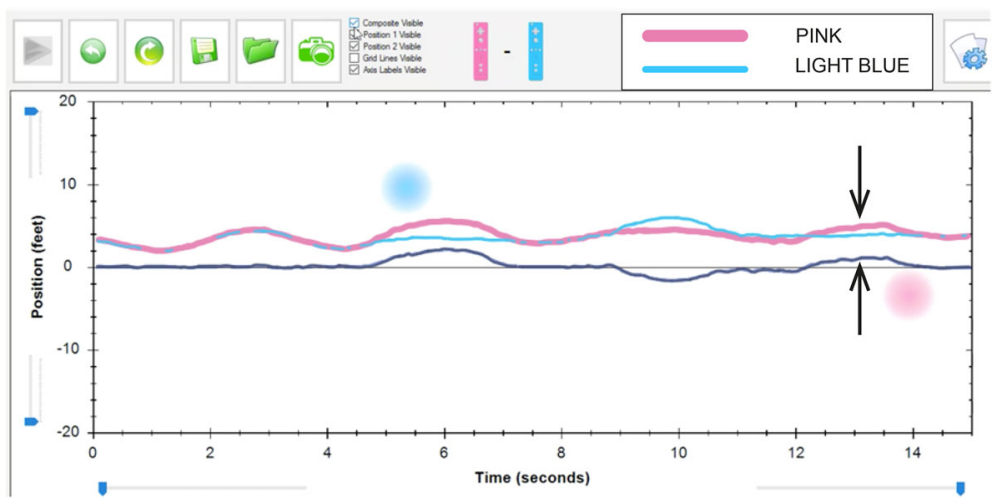

Fig. 9 Zev creates a new difference graph while speaking

We propose that semiosis is a key process in the formation of a general, which reflects the inherent presence of a continuum across which elements of a general communicate with each other. The graphs on the computer screen intermingle with the hand-held Wiimotes, such that "same level" and "same position" can refer to all of them and distribute qualities that remain distinct and, yet, overlapping.

Dan and Zev exchange the remotes. As soon as Zev grabs the remotes, he starts a new session and creates the graphs in Fig. 8:

18. Ricardo: So what do you think, how do you explain?

19. Zev: Well, every walk I've done checks, em... they have got a descending number and that's the distance of each control on the sensor [the LED bar] and then minuses the red one from the blue one [points towards the two remotes depicted on the top of the screen]. So if they are both the same [keeps the remotes next to each other], one minus one is zero, and the same with two minus two, so when we move them back and forwards the same [moves both remotes next to each other and starts a new session, beginning Fig. 9] it stays at zero, but when we move one [moves one remote backwards while he keeps the other one still, see the region around the arrows in Fig. 9].

20. Ricardo: So here, this minus this distance is zero [points to two overlapped points of the blue and pink lines, then to their difference graph on the $x$-axis] ... But here, what did it happen? [points to the two points of the pink and dark blue graphs marked by the arrows in Fig. 9].

21. Zev: Well, it's, it's different.

22. Ricardo: It's different... Alright, very good.

Commentary Zev begins Paragraph 19 by articulating three propositions: (1) "they've got a descending number," (2) "that's the distance of each control on the sensor," and (3) "then minuses the red one from the blue one." We will comment on the sense of each one and how they concatenate:

- They have got a descending number. 
"They" are, for the most part, the blue and pink graphs. Furthermore, since Zev had kept the two graphs going together, his saying " $a$ descending number" (singular) might suggest that both graphs descended by the same numbers. However, semiosis allows "they" to also relate to the hand-held Wiimotes, their icons on the computer screen, or the numbers implicit in the shape of the graphs. In the event, the numbers are descending if the remotes get closer to the LED bar. While such getting closer is an action undertaken by Zev, this proposition is articulated from the point of "them," so that they "got" a descending number. In other words, the descent is an effect passively undergone by the graph/number. The subsequent "and" signals the beginning of another proposition in the form of a juxtaposition, that is, the upcoming proposition is to be held in parallel with the previous one.

- That is the distance of each control [Wiimote] on the sensor [LED bar].

"That" brings up from the prior proposition a descending number to predicate of it that it is a specific distance between Wiimote and LED bar. This specification is inscribed in the general whose sense Zev is articulating: a general in which descending numbers, walking towards the LED bar, lowering graphs, and distances between Wiimotes and LED bar, are all mutually conditioned. The subsequent "and then" betokens an upcoming proposition that is not so much to be juxtaposed as coming after the prior ones.

- Then minuses the red one from the blue one.

After the numbers are gotten, they are "minused." Zev states that the red one is minused from the blue one. This can be understood in opposition to the equation depicted above the graphical space that appears as if blue is to be "minused" from red. However, the object of Zev's explanation is the case of red and blue numbers being equal, so that the result, zero, is indifferent with respect to which is minused from which.

In "one minus one is zero, and the same with two minus two," Zev uses particular examples to illustrate a general relationship. This is an instance of what Mason and Pimm (1984) have called "seeing the general in the particular." Zev is articulating a general that we could symbolize by: $\mathrm{A}-\mathrm{A}=0$; however, his understanding is far from being reducible to any formal definition: it encompasses countless qualities, such as the kinaesthesia of walking with two hands next to each other, the light blue and pink graphs going at the same height, the dark blue graph staying over the horizontal axes, the vast number of numbers that can be subtracted from themselves, the nothingness that remains after taking away_minusing — what had been given, or walking ahead as a kind of "descending." Navigating such boundless expansion of interrelated qualities is what we characterize as wayfaring a surplus of sensorimotor qualities towards grasping a general. Zev says that this general encompasses "when we move them back and forwards the same;" then he begins to point out, in words and gestures, that it excludes the case "when we move one..." and not the other one. This inclusion/exclusion criterion for a general that can be symbolized as A $-\mathrm{B}=0$ is reaffirmed by Zev ("it's different," Paragraph [21]), as he qualified the two cases pointed out by Ricardo in Paragraph [20]. 


\subsection{Keeping the difference graph above or below the $x$-axis}

23. Ricardo: So now we'll try to do something similar but keep the dark blue line always above. You can walk and move your hands, but keep the black, the dark blue line above the zero.

Mario comes to the front and holds the remotes. He starts a new session (Fig. 10a):

24. Ricardo: So now it's above [points to the dark blue graphs, around $10 \mathrm{~s}$ ].

Mario creates a new graph (Fig. 10b):

25. Ricardo: So what do you think? In order to get the dark blue line above the zero, what do you have to do?

26. Mario: Well, you, you make pi, pink bigger than blue so that...

27. Ricardo: ... the pink...

28. Mario: ... so you keep it above but if you wanted it below you have to have blue bigger than pink.

29. Ricardo: Ok, so let us have it, let us have it below now.

30. Mario: What?

31. Ricardo: Let us have it, the dark line, below.

Mario starts a new session, but very soon, he presses a button that generates again superimposed graphs. Refreshing the screen, Mario creates new graphs with a new session, starting with the pink remote in front, while the light blue one is kept farther from the LED bar (Fig. 11):

32. Ricardo: ... [while Mario is moving the light blue remote closer to the pink one] and then slowly you get it to zero. So what did you do to, to have it under the zero line?

33. Mario: I had to make the blue bigger than pink.

34. Ricardo: Ah!

35. Ricardo: So let us, eh, hand it to Dan, and so you create a pattern, you can do as many variations as you want, like walking and moving, but always keeping the blue line above the zero to start with...
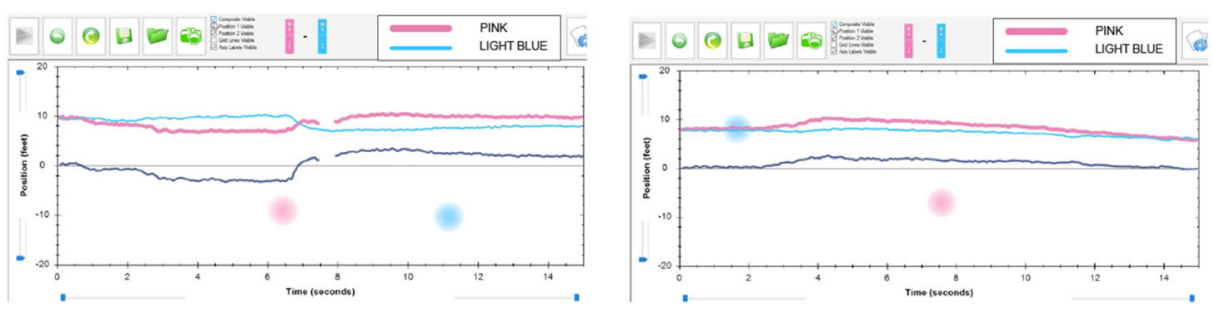

Fig. 10 a Mario creates a new difference graph. b He creates a difference graph above the $x$-axis 


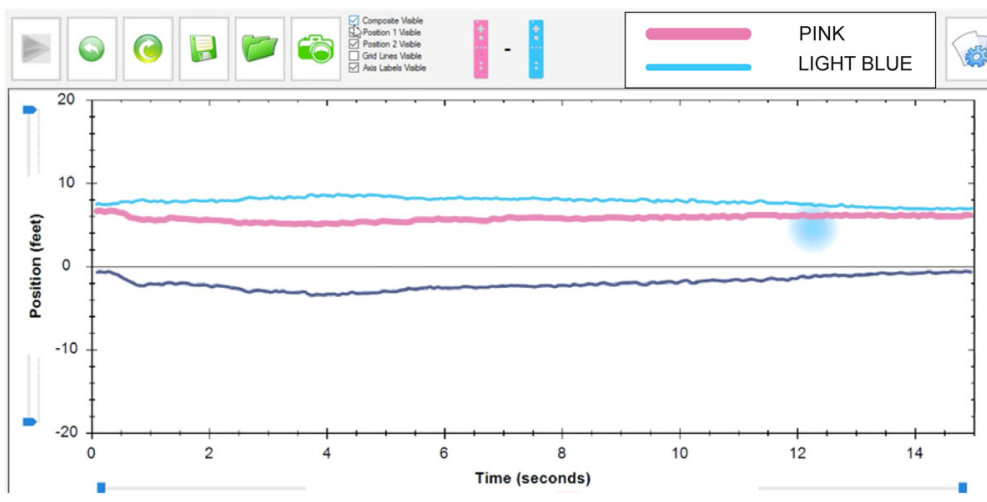

Fig. 11 Mario creates a new difference graph under the $x$-axis

Dan starts a new difference graph (Fig. 12a):

36. Ricardo: So what did you do, to keep it above?

37. Dan: Em, pink back and blue forward.

38. Ricardo: The pink back, further from this [pointing to the LED bar]. Em, and now keep it under this [the $x$-axis].

39. Dan: Oh! [restarts the session several times while Ricardo makes a few comments]

Dan creates the difference of Fig. $12 \mathrm{~b}$.

40. Ricardo: So what, what does it happen here, to get it below [the $x$-axis]?

41. Dan: You've to do the opposite... you put pink forward and the blue back.

42. Ricardo: Pink below, right? Ok. Great!

43. Ricardo: So now, Zev, do, do something like this: above, below. So first of all, but try to find out the variation, so what is it possible?

Zev is given the remotes and creates the graphs of Fig. 13a:

44. Ricardo: So, how did you change [the dark blue line] from below to above?

45. Zev: Em, by changing which controller was in front.

46. Ricardo: So which one was in front here? [points to the dark blue graph around the 4th second, where it is below the $x$-axis]

47. Zev: Em, [light] blue.
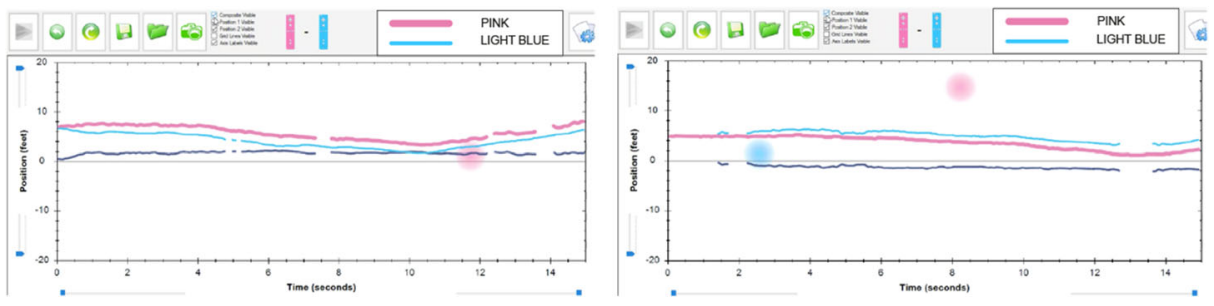

Fig. 12 a Dan creates a new difference graph above the $x$-axis. b Dan creates a new difference graph under the $x$ axis 

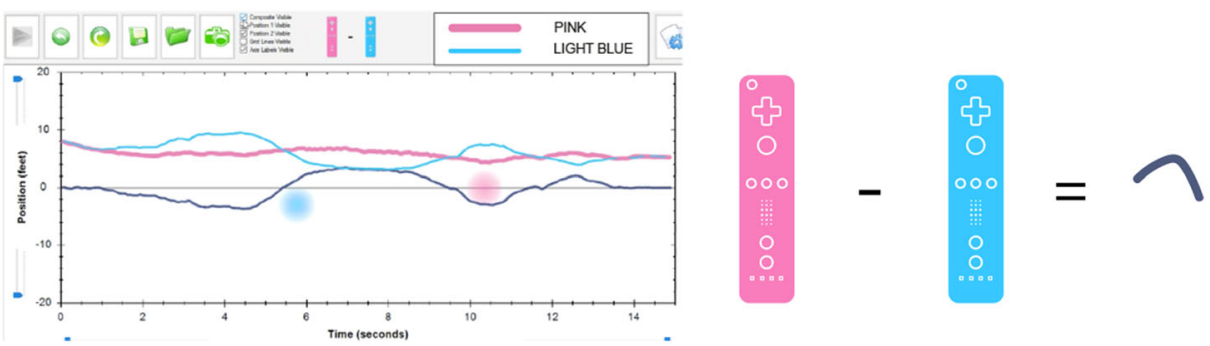

Fig. 13 a Zev generates a graph in which the difference graphs goes above and below zero. b Possible representation of the equation Zev discusses in Paragraph [49]

48. Ricardo: (...) And do you have a sense for why for the blue, for the dark blue line, to be below [the $x$-axis] then the pink has to be below [the light blue graph]?

49. Zev: Em, yep. Em, it's something to do with like maths and, like, because on there, it says the [seeming to point at image of the two Wiimotes on the screen] has been taken away and then it's hard to tell because it's not actual numbers, but, if you have more on one side, that will be a negative number... then, then, if you have them on the other side, it'll be a positive number, which is that [moves the dots on the screen along the dark blue line by controlling the remote].

Commentary In Sect. 4.2, Mario, Dan, and Zev characterized two complementary regions, one for the dark blue graph being above the $x$-axis and another for it being below. Mario separated these regions by contrasting "pink bigger than blue" and "blue bigger than pink" (Paragraphs [26] and [28]), Dan by "pink back and blue forward" or "pink forward and the blue back" (Paragraphs [37] and [41]), and Zev "by changing which controller was in front" (Paragraph [45]). In Sect. 2.3, we elaborated on a notion of semiosis as a drift of qualities across signifieds and signifiers. We also suggested the image of the interpretant as a continuum sustaining the "expansion, contraction and reproduction [of qualities] across signifieds and signifiers." The events transcribed in Sect. 4.2 inspire us to visualize compositional elements for the interpretant; namely, that instead of a single all-encompassing continuum, the interpretant would be akin to a Riemann surface with various sheets (see http://mathworld. wolfram.com/RiemannSurface.html). Mario, Dan, and Zev suggested several sheets: (1) a sheet hosting the dark blue graph with a region above the $x$-axis, distinct from a region below the $x$-axis; (2) a sheet hosting the pink and light blue graphs with regions separating which one is "bigger;" (3) a sheet containing the Wiimotes distributed along regions demarcating which one is closest or farthest from the LED bar; and (4) a sheet containing the Wiimotes distributed along regions that distinguish which one is in front of which one. The regions of each sheet map out with regions on the other sheets, such that, for example, "pink bigger than blue" in one sheet maps out with the "dark blue graph above $x$-axis" in another sheet. Figure 14 shows an attempt to illustrate such mapping across multiple sheets: a light trace goes across several pages of a book, allowing for certain regions on different sheets-pages to mutually exchange qualities. The children collectively unfolded semiosis as hosted by an expanding interpretant with multiple sheets, allowing for the mutual and multi-layered discrimination of diverse qualities, such as above/below, bigger/smaller, back/forward, and front/behind.

In Paragraph [49], Zev explained how the distinction between regions in some of these sheets relates to numbers. First, he points the remotes drawn on the computer screen above the 
Fig. 14 A light trace goes across several pages of a book, allowing for certain regions on each sheetpage to exchange qualities with others

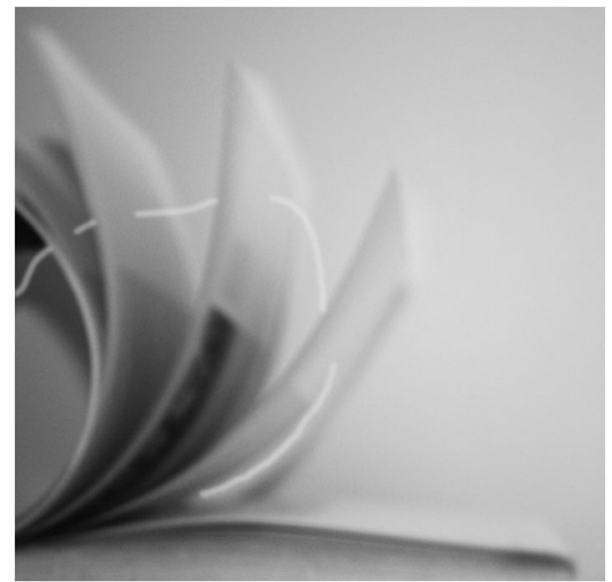

graphical region indicating that it "has been taken away," alluding to the subtraction displayed with the Wiimotes above the graphical space. Zev thinks that "it is hard to tell" what happens because the actual numbers are not shown. This latest remark makes a fleeting allusion to an unknown: beyond estimating possible values, the position numbers per se are not given. However, Zev quickly leaves behind these unknowns to distinguish two regions by their corresponding "sides:" more on one side of the minus will obtain a negative number, more on the other side a positive number. Zev described here a fifth sheet, which included the two Wiimotes depicted on the computer screen separated by a minus, with left and right sides or regions, such that having "more" on each side maps out numbers for the dark blue graph being positive or negative.

\section{Discussion}

During the selected episode, Mario, Dan, and Zev experimented with WiiGraph to make sense of a new graph - coloured dark blue - appearing on the computer screen. Through these experimentations, the children strived to attend and interpret a vast scope of sensible qualities, as we can infer from their words and actions, including not only all that was "there," such as shapes of three graphs mutually distinguished by colour, degrees of closeness to the monitor, moving dots that had to be kept inside the screen, signs of subtraction, unknown numbers, relative heights of graphical lines, a horizontal line sometimes called "zero," pink and blue Wiimotes with buttons on them, video cameras, synchronic movements of graphical lines from left to right, and more, but also all the kinaesthetic and proprioceptive qualities realized in the course of their movements and actions. The latter included the tonicity of muscular activity, moving hands while standing still, walking while keeping hands still, walking and moving hands cyclically, keeping hands next to each other, keeping one hand closer to the LED bar than the other one, bodily kinaesthetic responsiveness to events on the computer screen, changes on the computer screen responsive to their movements, going fast and slow, moving smoothly and abruptly, and more. Such abundance is what we refer to as a "surplus of sensible qualities." Over time, some of these qualities became more peripheral or more central than 
others. It was by virtue of kinaesthetic engagement that the graphs developed a temporality marked by complex and all-encompassing events, such as the graphs "going opposite" (Paragraph [6]), creating a "perfect zero" (Paragraph [12]), or getting "a descending number" (Paragraph [19]). Through kinaesthesia, signifiers and signifieds such as "level" and "position" (Paragraphs [14]-[17]), "forward/back" (Paragraph [41]), and "above/below" (Paragraph [45]), exchanged qualities by participating in the ongoing semiosis.

We think that navigating a surplus of sensible qualities is a critical aspect of an encounter and familiarization with a general, which in the present case study is one that can be symbolized by $\mathrm{A}-\mathrm{B}=\mathrm{C}$. What kind of navigation towards grasping a general across a surplus of sensorimotor qualities constitutes an abstraction? is the main question that we tried to address in this paper. Through the analysis of talk, gesture, and tool-use, we came to two intertwined processes appearing to characterize such navigation: semiosis and inhabiting an evocative and reckoning interpretant. The most central aspect of semiosis, we propose, is the exchange of qualities among signifiers and signifieds (e.g., descending number "moving closer" to the LED bar, Paragraph [19]). In semiosis, qualities move across a continuum (i.e., the interpretant) hosting signifieds and signifiers. We characterized this continuum as a Riemann surface with multiple sheets, each of which harbours regions (e.g., blue graph above or below the zero line) in mutual correspondence with others (e.g., pink Wiimote ahead or behind blue Wiimote). We summarize then, by saying that abstracting a general entails familiarity with a layered continuous interpretant enabling the active exchange of sensible qualities while keeping them distinct and communicating. It is through this dynamic exchange, which involves proprioceptive and kinaesthetic activity with the instrument, that the encounter with $\mathrm{A}-\mathrm{B}=\mathrm{C}$ occurs and that activity becomes relevant in approaching early algebra, as a way of mobilizing variables and equations through the body while opening up a range of possibilities for semiosis.

Beyond early algebra, we end this discussion by elaborating on the broader theme of this paper, which is the nature of mathematical abstraction, especially as it pertains the grasping of generals. Noble, Nemirovsky, Wright, and Tierney (2001) describe a useful example regarding the concept of one half.

[How does] one understands the familiar idea of one half? Try out these two tasks yourself. First, find one half of the quantity 3275 and write your answer. Next, walk across a room once, and then walk across the room again but try to walk half as fast as you did the first time. (...) Dividing 3275 in half most likely drew on your computational ability and your number sense, causing you to think about one half as a relation between two numbers. Walking across the room half as fast as before probably caused you to think about how to qualitatively compare one or more of the quantities speed, distance, and time when comparing your two motions across the room. You may also have tried to feel the quantities of speed or time in your body as you made the motion itself. (...). Although one may be tempted to look for the essential element of "halfness" in each of these experiences and to try to find ways to give students access to this element, one's own sense of halfness comes from these experiences and many others like them. (pp. 105-106)

What is abstract about a general, be it $1 / 2$ or $\mathrm{A}-\mathrm{B}=\mathrm{C}$, is not something extracted and isolated from perception, materiality, and motor activity (e.g., an idea encapsulated by a formal definition) but, on the contrary, a type of navigation that is immanent to an infinite realm of sensorimotor qualities that living beings follow up on, with the fallible but indispensable orientation of instruments, diagrams, and skilful engagements with them. 
Acknowledgements The authors wish to thank the important feedback provided by three anonymous reviewers and Arthur Bakker, and also Vinay Kathotia and Dragan Trninic who commented on a previous draft of this manuscript. Chiara Ferrari gave us invaluable graphic design support for the improvement of the figures. Development of WiiGraph was supported in part by the InforMath project, funded by the National Science Foundation through grant DRL-1323587. All opinions and analysis expressed herein are those of the authors and do not necessarily represent the position or policies of the Foundation.

Open Access This article is licensed under a Creative Commons Attribution 4.0 International License, which permits use, sharing, adaptation, distribution and reproduction in any medium or format, as long as you give appropriate credit to the original author(s) and the source, provide a link to the Creative Commons licence, and indicate if changes were made. The images or other third party material in this article are included in the article's Creative Commons licence, unless indicated otherwise in a credit line to the material. If material is not included in the article's Creative Commons licence and your intended use is not permitted by statutory regulation or exceeds the permitted use, you will need to obtain permission directly from the copyright holder. To view a copy of this licence, visit http://creativecommons.org/licenses/by/4.0/.

\section{References}

Abrahamson, D., \& Sánchez-García, R. (2016). Learning is moving in new ways: The ecological dynamics of mathematics education. Journal of the Learning Sciences, 25(2), 203-239.

Baccaglini-Frank, A., \& Mariotti, M. A. (2010). Generating conjectures in dynamic geometry: The maintaining dragging model. International Journal of Computers for Mathematical Learning, 15(3), 225-253.

Blanton, M., Brizuela, B., Stephens, A., Knuth, E., Isler, I., Gardiner, A., ... Stylianou, D. (2018). Implementing a framework for early algebra. In C. Kieran (Ed.), Teaching and learning algebraic thinking with 5- to 12year-olds: The global evolution of an emerging field of research and practice. ICME-13 monographs (pp. 27-49). Springer International: Cham, Switzerland.

Bodanskii, F. (1969/1991). The formation of an algebraic method of problem solving in primary school children. In V. Davydov (Ed.), Soviet studies in mathematics education: Psychological abilities of primary school children in learning mathematics (vol. 6, pp. 275-338). Reston, VA: The National Council of Teachers of Mathematics.

Carraher, D. W., Schliemann, A. D., Brizuela, B., \& Earnest, D. (2016). Arithmetic and algebra in early mathematics education. In E. A. Silver \& P. A. Kenney (Eds.), More lessons learned from research (vol. 2, pp. 109-122). Reston, VA: NCTM.

Clements, D. H. (2000). 'Concrete' manipulatives, concrete ideas. Contemporary Issues in Early Childhood, $1(1), 45-60$.

Coles, A., \& Sinclair, N. (2018). Re-thinking 'concrete to abstract': Towards the use of symbolically structured environments. In M. Ö. E. Bergqvist, C. Granberg, \& L. Sumpter (Eds.), Proceedings of the 42nd conference of the International Group for the Psychology of mathematics education (vol. 2, pp. 275-282). Umeå, Sweden: PME.

Davydov, V. V. (1990). Soviet studies in mathematics education (vol. 2). Reston, VA: National Council of Teachers of Mathematics.

de Freitas, E., Ferrara, F., \& Ferrari, G. (2019). The coordinated movements of collaborative mathematical tasks: The role of affect in transindividual sympathy. ZDM Mathematics Education, 51(2), 305-318.

Deleuze, G. (1990). The logic of sense. New York, NY: Columbia University Press.

Dreyfus, T. (2014). Abstraction in mathematics education. In S. Lerman (Ed.), Encyclopedia of mathematics education (pp. 5-8). Dordrecht, The Netherlands: Springer.

Duijzer, C., Van den Heuvel-Panhuizen, M., Veldhuis, M., Doorman, M., \& Leseman, P. (2019). Embodied learning environments for graphing motion: A systematic literature review. Educational Psychology Review, 31(3), 597-629.

Frege, G. (1980). On sense and reference. In P. Geach \& M. Black (Eds.), Translations from the philosophical writings of Gottlob Frege. Oxford, UK: Blackwell.

Hershkowitz, R., Schwarz, B. B., \& Dreyfus, T. (2001). Abstraction in context: Epistemic actions. Journal for Research in Mathematics Education, 32(2), 195-222.

Kaput, J. J. (2008). What is algebra? What is algebraic reasoning? In J. J. Kaput, D. Carraher, \& M. Blanton (Eds.), Algebra in the early grades. New York, NY: Lawrence Erlbaum. 
Kaput, J. J., Blanton, M. L., \& Moreno, L. (2008). Algebra from a symbolization point of view. In J. J. Kaput, D. W. Carraher, \& M. L. Blanton (Eds.), Algebra in the early grades (pp. 19-55). New York, NY: Lawrence Erlbaum.

Kieran, C., Pang, J., Schifter, D., \& Fong Ng, S. (2016). Early algebra. Research into its nature, its learning, its teaching. Cham, Switzerland: Springer.

Mason, J., \& Pimm, D. (1984). Generic examples: Seeing the general in the particular. Educational Studies of Mathematics, 15(3), 277-289.

Nemirovsky, R., Kelton, M., \& Rhodehamel, B. (2012). Gesture and imagination: On the constitution and uses of phantasms. Gesture, 12(2), 130-165.

Nemirovsky, R., Kelton, M., \& Rhodehamel, B. (2013). Playing mathematical instruments: Emerging perceptuomotor integration with an interactive mathematics exhibit. Journal of Research in Mathematics Education, 44(2), 372-415.

Nemirovsky, R., Tierney, C., \& Wright, T. (1998). Body motion and graphing. Cognition and Instruction, 16(2), $119-172$.

Ng, S. F., \& Lee, K. (2009). The model method: Singapore children's tool for representing and solving algebraic word problems. Journal for Research in Mathematics Education, 40(3), 282-313.

Noble, T., Nemirovsky, R., Wright, T., \& Tierney, C. (2001). Experiencing change: The mathematics of change in multiple environments. Journal for Research in Mathematics Education, 32(1), 85-108.

Noss, R., Hoyles, C., \& Pozzi, S. (2002). Abstraction in expertise: A study of nurses' conceptions of concentration. Journal for Research in Mathematics Education, 33(3), 204-229.

Peirce, C. S. (1931-1935). The Collected Papers of Charles Sanders Peirce. Electronic edition reproducing Vols. I- VI [C. Hartshorne \& P. Weiss (eds.), Cambridge: Harvard University Press, I93I-I935]; Vols. VIIVIII [A. W. Burks (Ed.), same publisher, 1958]. Charlottesville: Intelex Corporation. (Quoted as CP, followed by volume and paragraph.).

Radford, L. (2014). The progressive development of early embodied algebraic thinking. Mathematics Education Research Journal, 26(2), 257-277.

Roth, W. M., \& Hwang, S. (2006). On the relation of abstract and concrete in scientists' graph interpretations: A case study. The Journal of Mathematical Behavior, 25(4), 318-333.

Serres, M. (2017). Geometry. The third book of foundations. London, UK: Bloomsbury Academic.

Sinclair, N., \& Yurita, V. (2008). To be or to become: How dynamic geometry changes discourse. Research in Mathematics Education, 10(2), 135-150.

Straesser, R. (2002). Cabri-Géomètre: Does dynamic geometry software (DGS) change geometry and its teaching and learning? International Journal of Computers for Mathematical Learning, 6(3), 319-333.

Verillon, P., \& Rabardel, P. (1995). Cognition and artifacts: A contribution to the study of thought in relation to instrumented activity. European Journal of Psychology of Education, 10(1), 77-101.

Wilensky, U. (1991). Abstract meditations on the concrete and concrete implications for mathematics education. In I. Harel \& S. Papert (Eds.), Constructionism (pp. 193-204). Norwood, NJ: Ablex publishing.

Publisher's note Springer Nature remains neutral with regard to jurisdictional claims in published maps and institutional affiliations. 